\title{
Syringotropic mycosis fungoides responding well to VELP chemotherapy: A case report
}

\author{
YANG LUO, LI ZHANG, YU-JIAO SUN, HUA DU and GUI-LAN YANG
}

\begin{abstract}
Department of Dermatology, Lanzhou General Hospital of Lanzhou Military Region, Lanzhou, Gansu 730050, P.R. China
\end{abstract}
Received December 31, 2014; Accepted February 18, 2016

DOI: 10.3892/etm.2016.3183

\begin{abstract}
Mycosis fungoides (MF), a low-malignant lymphoproliferative disorder, is the most common type of cutaneous T-cell lymphoma. The current study reported a case of syringotropic $\mathrm{MF}$, a rare variant of $\mathrm{MF}$, which presented with reactive $\mathrm{B}$ cell proliferation, lymphoid follicle formation, hair loss and lymphadenopathy. The clinical manifestations of the patient were MF-like lumps. Immunohistochemical staining of AE1/AE3 showed that there were abundant infiltrated lymphocytes surrounding the syringocystadenoma. In addition, the direction of the lymphocyte arrangement was consistent with the meandering direction of syringocystadenoma. The patient did not respond to 1-month narrowband (311-nm) ultraviolet therapy; however, a good response was obtained subsequent to one cycle of chemotherapy with vincristine sulfate, etoposide, L-asparaginase and prednisone acetate (know as the VELP regimen). After 7 days of VELP chemotherapy, the skin lesions were ameliorated, hair loss was improved and lymphadenopathy disappeared. No lymphadenopathy or new skin lesions were observed during 6 months of follow-up.
\end{abstract}

\section{Introduction}

Mycosis fungoides (MF) is a low-grade lymphoproliferative disorder, and the most common MF type is cutaneous T-cell lymphoma, accounting for $50 \%$ all cutaneous T-cell lymphoma (1). Syringotropic MF (SMF) is a rare variant of MF (2), which is defined as MF with infiltration of eccrine glands by neoplastic cells, according to the World Health Organization classification (3). Typical disease characteristics are patches, plaques, nodular masses, papules, scales and lichenification (2). The shapes of the skin lesions are often round, circular or irregular, the color is often red, purple red or dark brown, and the lesions are typically accompanied

Correspondence to: Dr Yang Luo, Department of Dermatology, Lanzhou General Hospital of Lanzhou Military Region, 333 Binhenan Road, Lanzhou, Gansu 730050, P.R. China

E-mail: lytmmu@163.com

Key words: syringotropic mycosis fungoides, VELP chemotherapy, alopecia by pruritus or baldness (2). Early-stage SMF is treated with narrowband $(311 \mathrm{~nm})$ ultraviolet therapy (2). To date, only a limited number of SMF cases have been reported in the literature $(4,5)$.

According to the current guidelines, the syringotropic and folliculotropic forms of the disease are classified as adnexotropic MF $(4,6)$. Solitary, erythematous, punctate and anhidrotic patches are often clinically observed in the syringotropic form $(4,6)$. Currently, the main recommended treatments for SMF are extracorporeal phototherapy and total skin electron beam therapy $(2,5,7)$. The present study reported a case of rapidly progressive SMF with alopecia and lymphadenopathy. A good response was observed following one cycle of chemotherapy with vincristine sulfate, etoposide, L-asparaginase and prednisone acetate, known as the VELP chemotherapy regimen.

\section{Case report}

In May 2014, a 19-year-old man presented at Lanzhou General Hospital (Lanzhou, China), complaining of systemic infiltrative dark red skin plaques with itching that persisted for 5 months. At 5 months prior to the presentation at Lanzhou General Hospital, the patient had developed red plaques with itching on the trunk. The number of skin lesions increased gradually and they were dispersed across the entire body, being particularly prominent on the head and trunk. Physical examination revealed stable vital signs and enlarged bilateral submandibular, neck and armpit lymph nodes. The lymph nodes were soybean-sized, and had a tenacious texture, moderate mobility and no evident tenderness.

Cardiac, pulmonary and abdominal examinations revealed no marked abnormalities. A skin examination revealed skin lesions scattered over the entire body, which were particularly prominent on the head, trunk and lower extremities. The lesions were manifested as dark red infiltrative plaques and tumors, with diameters of 1-4 cm, clear boundaries and a hard texture. Certain skin lesions were ulcerated, and there were clear ulcerative exudates on the trunk. The patient suffered from alopecia, which was particularly evident on the occipital scalp (Fig. 1A-C).

The patient underwent skin biopsy twice within a period of 2 months; the first was performed on admission to hospital and the second biopsy was performed as a diagnosis could not be confirmed. Biopsy tissues were fixed in $10 \%$ formalin 

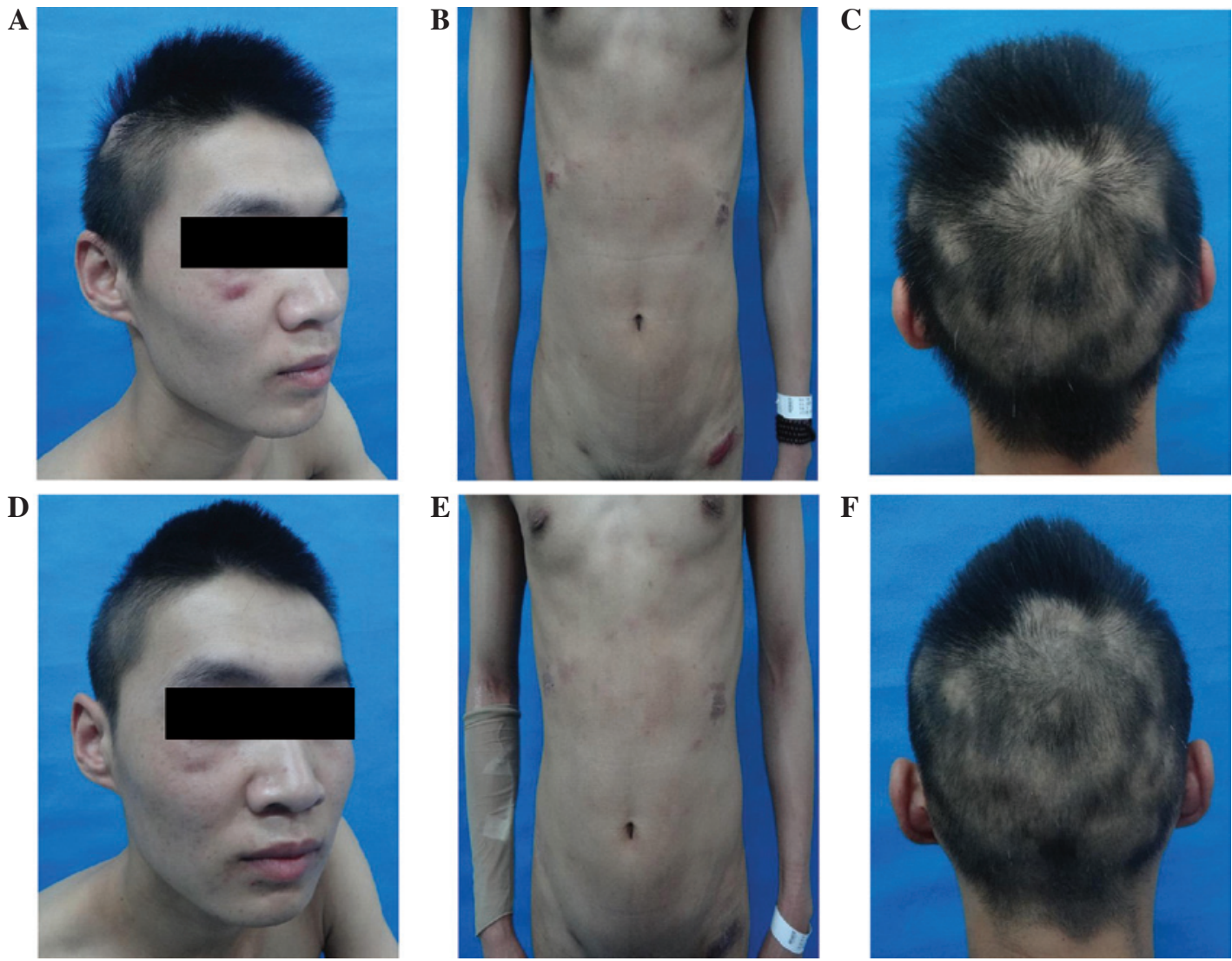

Figure 1. Photographs of the patient. (A) Front view of the head, (B) front view of the trunk, and (C) back view of the head, prior to VELP chemotherapy. (D) Front view of the head, (E) front view of the trunk, and (F) back view of the head, subsequent to VELP chemotherapy. The VELP regimen involved administration of vincristine sulfate, etoposide, L-asparaginase and prednisone acetate.

(Shanghai Baoman Biotechnology Co., Ltd., Shanghai, China) for $12 \mathrm{~h}$, then dehydrated and embedded in paraffin (Shanghai Hualing Equipment Factory, Shanghai, China) and sectioned $(4 \mu \mathrm{m})$ using a Leica section station (RM2016; Leica Microsystems GmbH, Wetzlar, Germany). The sections were then bleached using a Pathological Bleaching and Drying Machine (Changzhou Electronic Instrument Co., Ltd., Changzhou, China), dewaxed and stained with hematoxylin (Shanghai Chemical Reagent Co., Ltd., Tianjin, China) and eosin (HE; Third Factory of Shanghai Reagent Chemicals, Shanghai, China) for $15 \mathrm{~min}$. The HE-stained sections were observed with an optical microscope (BX53; Olympus Corporation, Tokyo, China) and diagnosed by a pathologist. Histopathological examination revealed mild epidermal spongiosis, unremarkable lymphocyte epidermotropism, abundant infiltration of lymphocytes into the dermis, no obvious heterotypic lymphocytes, granulomas formed by epithelioid cells and macrophages in the dermis (Fig. 2A and B), and abundant lymphocyte infiltration around the metaplastic glands (Fig. 2C and D). There was abundant lymphocyte infiltration around hair follicles. Nodule-like structures, abundant macrophages and lymphoid follicle formation were visible in the lower dermis (Fig. 2E and F).

Biopsy tissues underwent immnohistochemical staining as follows: Sections (1.5 $\mathrm{cm} \times 1.5 \mathrm{~cm} \times 4 \mu \mathrm{m})$ were cut (RM2235; Leica Microsystems $\mathrm{GmbH}$ ), dewaxed in xylene, boiled in $0.01 \mathrm{~mol} / \mathrm{l}$ citrate buffer ( $\mathrm{pH}$ 9.0) (both purchased from Beijing Zhongshan Jinqiao Biotechnology Co., Ltd., Beijing, China) for 3 min, cooled and washed with phosphate buffer solution (PBS) 3 times for $3 \mathrm{~min}$, incubated with $3 \% \mathrm{H}_{2} \mathrm{O}_{2}$ at $42^{\circ} \mathrm{C}$ for $10 \mathrm{~min}$, washed and incubated in PBS for $5 \mathrm{~min}$ at $37^{\circ} \mathrm{C}$, and incubated with the following primary antibodies at $37^{\circ} \mathrm{C}$ for $1 \mathrm{~h}$ : Monoclonal mouse anti-AE1/AE3 (1:100 dilution; IR053), monoclonal mouse anti-Bcl6 (1:50 dilution; IR625), polyclonal rabbit anti-CD3 (1:100 dilution; IR503), monoclonal mouse anti-CD4 (1:100 dilution; IR649), monoclonal mouse anti-CD8 (1:150 dilution; IR623), monoclonal mouse anti-CD20 (1:100 dilution; IR604), monoclonal mouse anti-CD30 (1:50 dilution; IR602), monoclonal mouse anti-CD68 (1:200; IR613) and monoclonal mouse anti-Ki67 (1:100; IR626) (all purchased from Dako, Glostrup, Denmark). The sections were washed for 5 min with PBS twice, incubated with biotinylated goat anti-rabbit/mouse polyclonal secondary antibody (1:500 dilution; K0675; Dako) at $37^{\circ} \mathrm{C}$ for $30 \mathrm{~min}$, and washed for 5 min with PBS twice. The sections were then incubated with diaminobenzidine solution (Beijing Zhongshan Jinqiao Biotechnology Co., Ltd.) at $37^{\circ} \mathrm{C}$ for $5 \mathrm{~min}$, washed with distilled water for $1 \mathrm{~min}$, stained with hematoxylin for $1 \mathrm{~min}$ and washed with distilled water for $1 \mathrm{~min}$. The sections were incubated in hydrochloric acid ethanol solution for $10 \mathrm{sec}$, washed with distilled water for $1 \mathrm{~min}$ and sealed. The sections were then observed under an Olympus BX53 microscope (Olympus Corporation). Immunohistochemical tests were positive for $\mathrm{CD} 3$ and $\mathrm{CD} 4$ and negative for $\mathrm{CD} 8$ in the newly formed T lymphocytes (Fig. 3A). In addition, the analysis revealed an abundance of CD3-positive lymphocytes 

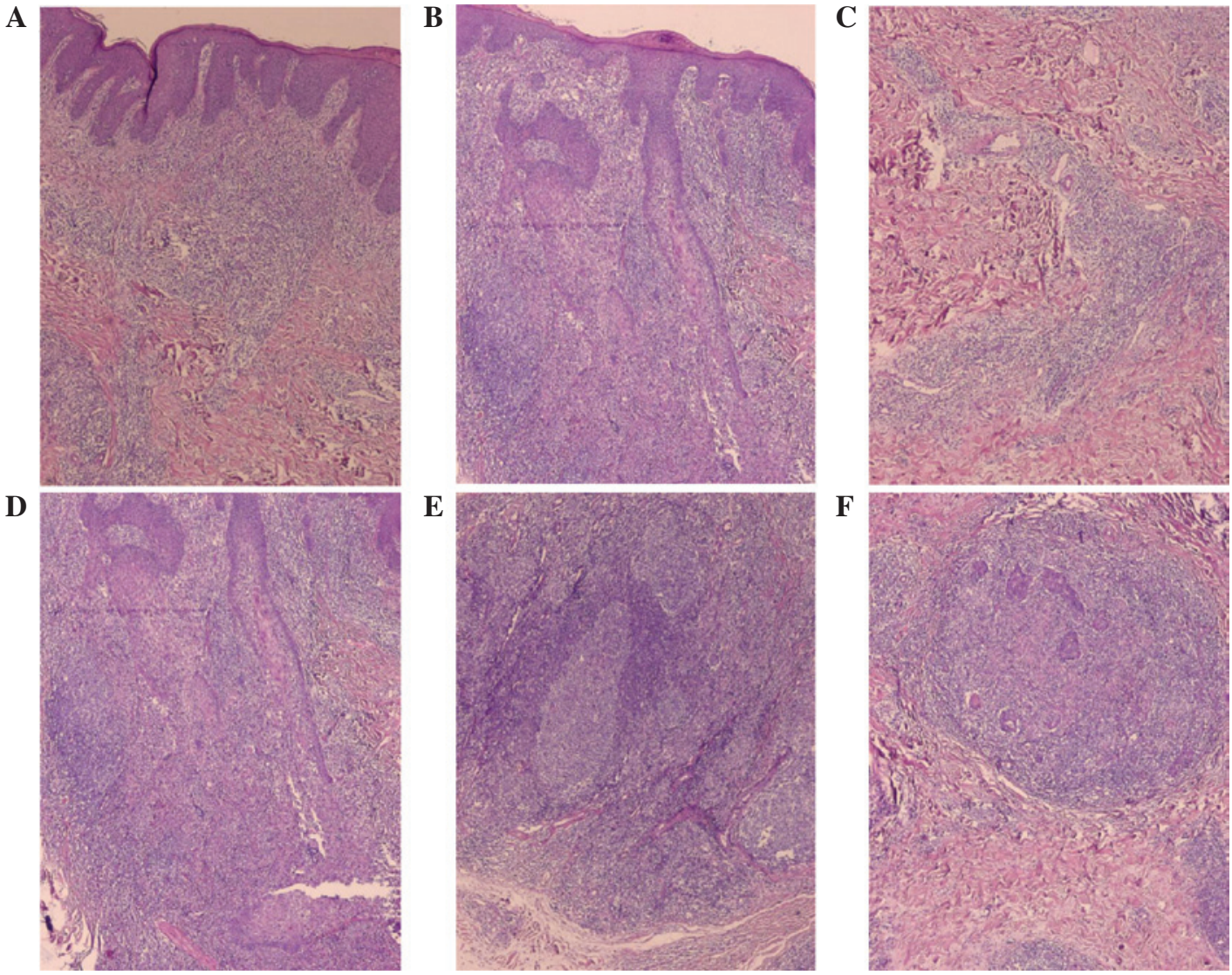

Figure 2. Hematoxylin-eosin staining of the second skin biopsy. (A and B) Epidermis and dermis; (C) metaplastic sweat glands in the dermis; (D) hair follicles; and deep dermis at magnifications of (E) x40 and (F) x100. Original magnification for (A-E), x40.

A

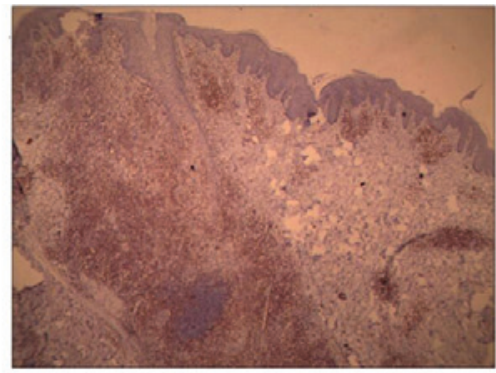

D

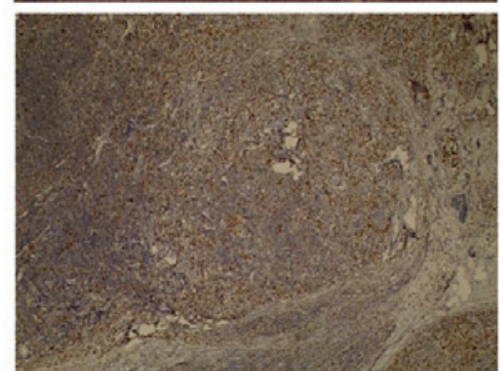

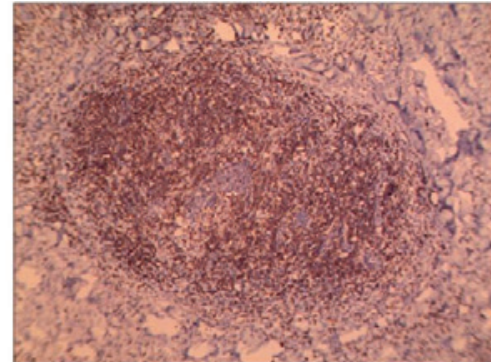

$\mathbf{E}$

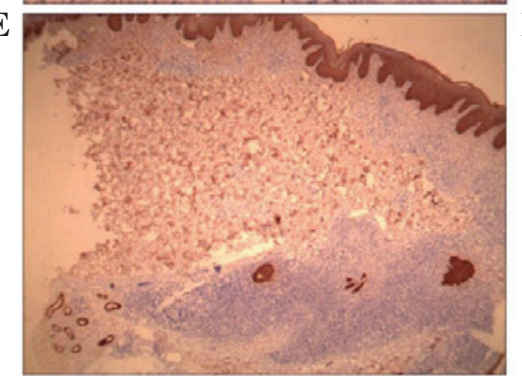

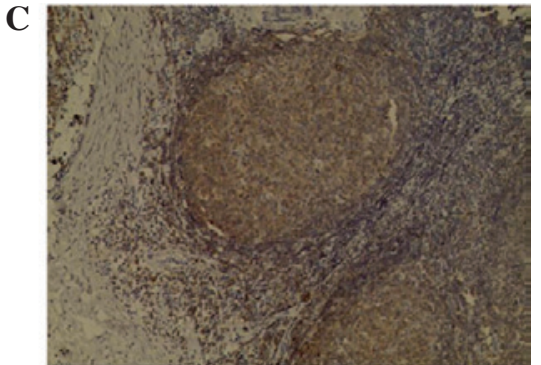

F

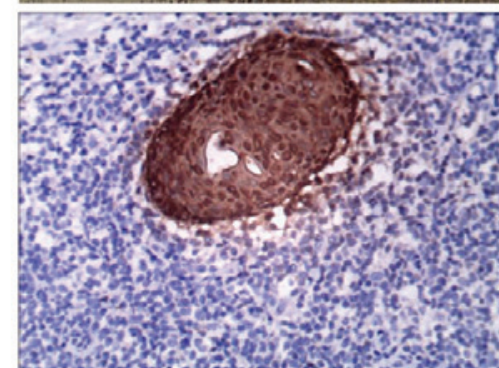

G

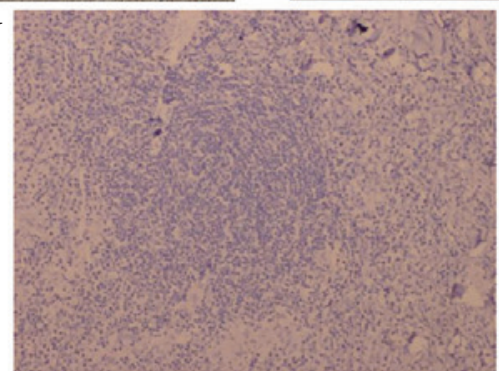

$\mathbf{H}$

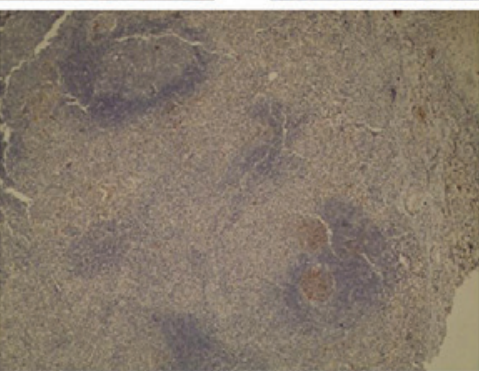

Figure 3. Immunohistochemical staining of the skin biopsy. CD3 at magnifications of (A) x40 and (B) x100; (C) CD20 (magnification, x100); (D) CD68 (magnification, x100); cytokeratin AE1/AE3 at magnifications of (E) x40 and (F) x200; (G) CD30 (magnification, x100); and (H) CD1a (magnification, x40). 
infiltrating around the hair follicles. CD3-positive lymphocytes (Fig. 3B), abundance of CD20-positive B cells (Fig. 3C), and a small quantity of CD68-positive cells in the nodule-like structures (Fig. 3D) were also observed. The accumulating infiltrative lymphocytes were negative for CD30 (Fig. 3E), S-100 and CD1a (Fig. 3F). Cytokeratin AE1/AE3 staining showed an abundance of lymphocytes infiltrating around brown-staining sweat ducts and arrangement of lymphocytes coinciding with the coiling direction of the sweat ducts, while an abundance of infiltrated lymphocytes was observed around the metaplastic eccrine glands (Fig. 3G and H).

Acid-fast staining was performed as follows: Samples were dewaxed and stained with $5 \%$ alkaline fuchsin solution (diluted in $100 \%$ ethanol; Tianjin Zhiyuan Chemical Reagent Co., Ltd., Tianjin, China) for $5 \mathrm{~min}$, heated to $\sim 100^{\circ} \mathrm{C}$ and washed with distilled water. Then, $3 \%$ hydrochloric acid and ethanol solution was added until the sections were no longer faded by the water, and the samples were double staining with $0.5 \%$ methylene blue solution (Hangzhou Baisi Biotechnology Co., Ltd., Hangzhou, China) for 30-60 sec, washed with distilled water and air-dried. The samples were then examined under an optical microscope (BX53; Olympus Corporation). The background of the stained sections was blue, and no red-stained acid-fast bacillus was observed, therefore excluding leprosy as a diagnosis.

Based on the aforementioned findings, SMF was diagnosed, and the clinical stage of the disease was determined as IIB (8). Subsequently, the patient received narrowband $(311 \mathrm{~nm})$ ultraviolet therapy (SS06 Phototherapy Unit; Sigma-Aldrich, St. Louis, MO, USA) for 1 month; however, the skin lesions were not improved and new skin lesions appeared, with enlargement of the neck, armpit and submandibular lymph nodes also observed. The patient was then administered VELP chemotherapy (9). The VELP chemotherapy regimen was as follows: $2 \mathrm{mg} /$ day intravenous vincristine sulfate (Zhejiang Hisun Pharmaceutical Co., Ltd., Taizhou, China) for 1 day; $0.1 \mathrm{~g} /$ day intravenous etoposide (Jiangsu Hengrui Medicine Co., Ltd., Jiangsu, China) for 1 day; 10,000 U/day intravenous L-asparaginase (Changzhou Qianhong Biochemical Pharmaceutical Co., Ltd., Changzhou, China) for 6 days; and $5 \mathrm{mg} /$ day oral prednisone acetate (Tianjin Lisheng Pharmaceutical Co. Ltd., Tianjin, China), three times per day for 7 days. After 7 days of VELP chemotherapy, the skin lesions were clearly ameliorated, the lesion color became darker, and hair loss was decreased (Fig. 1D-F). No new skin lesions appeared during the 6 months of follow-up; skin lesions were recovered and hair was regrown.

Written informed consent was obtained from the patient for the publication of the present case study.

\section{Discussion}

$\mathrm{MF}$, a low-malignant $\mathrm{T}$ lymphoma originating from the skin, is the most common type of skin $\mathrm{T}$ lymphoma, accounting for $50 \%$ of all the skin lymphomas (3). Beside from typical $\mathrm{MF}$, there are numerous clinical and pathological variants, including folliculotropic MF (10), SMF $(2,6,7,11)$ and granulomatous MF (12). The present case is a rare SMF with reactive $B$ cell proliferation and lymphoid follicle formation (5).
SMF was first described by Sarkany (13) in 1969, after which, reports of SMF have increased gradually. For instance, in 2011, Pileri et al (14) reported 14 cases of SMF. Clinically, the major symptoms of SMF are skin patches, plaques, nodule-like masses, papules, scales and lichenification. The skin lesions may be round, circular or irregular in shape, and are frequently red, dark red or dark brown in color. The lesions are frequently accompanied by pruritus and occasionally by alopecia $(2,4)$. Santucci et al $(15)$ proposed that the presence of medium-large cerebriform cells in the epidermis or in clusters in the dermis is a highly reliable feature for early diagnosis of MF. SMF is histologically characterized by infiltration of the eccrine glands by atypical lymphocytes in association with syringolymphoid hyperplasia.

The current patient showed mild epidermal spongiosis, unremarkable lymphocyte epidermotropism, massive lymphocyte infiltration in the dermis, no heterotypical lymphocytes, granulomas formed by epithelioid cells and macrophages in the dermis, and evident lymphocyte infiltration around the metaplastic glands. In addition, abundant lymphocyte infiltration was observed around the hair follicles. Immunohistochemical examination showed CD3/CD4 positivity and CD8 negativity in newly formed T lymphocytes, and CD3-positive lymphocyte infiltration around the hair follicles. Furthermore, CD3-positive lymphocytes, CD20-positive B cells and CD68-positive cells were also observed in the nodule-like structures. Accumulating infiltrative lymphocytes were negative for CD30, S100 and CD1a. Cytokeratin AE1/AE3 staining revealed abundant lymphocyte infiltration around the brown-staining sweat ducts and abundant lymphocyte infiltration around the metaplastic eccrine glands. Based on these findings, a diagnosis of SMF with B cell proliferation and lymphoid follicle formation was established (5).

Subsequent to reviewing a total of $23 \mathrm{SMF}$ cases in the literature using PubMed (http://www.ncbi.nlm.nih.gov/pubmed; keywords, syringotropic mycosis fungoides; date of search, December 2015), the disease often occurred in young people, particularly individuals with an age of approximately 20 years (2). In the current case, the patient was 19 years old. The patient presented pruritus, and leprosy was excluded based on histopathological examination, immunohistochemical studies and acid-fast staining. Subsequently, the patient was diagnosed with SMF, which is reported to be responsive to phototherapy (16). Therefore, the patient was initially given 311-nm ultraviolet therapy for 1 month; however, the skin lesions were not mitigated and new skin lesions appeared, with neck, armpit and submandibular lymph nodes becoming larger.

A number of studies have shown that radiotherapy achieved an appreciable efficacy in the treatment of $\operatorname{SMF}(2,17)$. However, the patient refused radiotherapy, and thus VELP chemotherapy was administered. After 7 days of chemotherapy, the skin lesions clearly remitted and hair loss was improved. During 6 months of follow-up, no new skin lesions or lymphadenopathy were observed, which suggested a good response to VELP chemotherapy. To the best of our knowledge, no studies have reported the use of VELP chemotherapy for the treatment of SMF thus far. Therefore, the present case provides novel findings for the use and efficacy of VELP chemotherapy in SMF patients with lymphadenopathy. However, the underlying 
mechanism for the efficacy of VELP chemotherapy for SMF remains unknown and requires further investigation.

In conclusions, the present study reported a case of SMF with reactive $\mathrm{B}$ cell proliferation, lymphoid follicle formation, hair loss and lymphadenopathy. The patient did not respond to 311-nm ultraviolet treatment; however, a good response to VELP chemotherapy was observed. VELP chemotherapy significantly improved the symptoms, and no new symptoms were observed during the 6-month follow-up period.

\section{References}

1. Nashan D, Faulhaber D, Ständer S, Luger TA and Stadler R: Mycosis fungoides: A dermatological masquerader. $\mathrm{Br}$ J Dermatol 156: 1-10, 2007.

2. de Masson A, Battistella M, Vignon-Pennamen MD, Cavelier-Balloy B, Mouly F, Rybojad M, Bouaziz JD, Petit A, Saussine A, Ronceray S, et al: Syringotropic mycosis fungoides: Clinical and histologic features, response to treatment and outcome in 19 patients. J Am Acad Dermatol 71: 926-934, 2014

3. Burg G, Kempf W, Cozzio A, Feit J, Willemze R, S Jaffe E, Dummer R, Berti E, Cerroni L, Chimenti S, et al: WHO/EORTC classification of cutaneous lymphomas 2005: Histological and molecular aspects. J Cutan Pathol 32: 647-674, 2005.

4. Bakar O, Seckin D, Demirkesen C, Baykal C and Buyukbabani N: Two clinically unusual cases of folliculotropic mycosis fungoides: One with and the other without syringotropism. Ann Dermatol 26: 385-391, 2014.

5. Wang L, Wang G and Gao T: Granulomatous syringotropic mycosis fungoides with two lesions having reactive $\mathrm{B}$-cell proliferation. J Cutan Pathol 41: 400-406, 2014

6. Willemze R, Jaffe ES, Burg G, Cerroni L, Berti E, Swerdlow SH, Ralfkiaer E, Chimenti S, Diaz-Perez JL, Duncan LM, et al: WHO-EORTC classification for cutaneous lymphomas. Blood 105: 3768-3785, 2005

7. Jennings L, Campbell SM, Yaar R, Mahalingam M, Sahni D, Lerner A and Rünger TM: Generalized syringotropic mycosis fungoides responsive to extracorporeal photopheresis. $\mathrm{Br}$ J Dermatol 170: 200-202, 2014.
8. Trautinger F, Knobler R, Willemze R, Peris K, Stadler R, Laroche L, D'Incan M, Ranki A, Pimpinelli N, Ortiz-Romero $\mathrm{P}$, et al: EORTC consensus recommendations for the treatment of mycosis fungoides/Sézary syndrome. Eur J Cancer 42: 1014-1030, 2006.

9. Pectasides D, Aravantinos G, Visvikis A, Bakoyiannis C, Halikia A, Kalofonos C, Kosmidis P, Skarlos D and Fountzilas G: Platinum-based chemotherapy of primary extragonadal germ cell tumours: The hellenic cooperative oncology group experience. Oncology 57: 1-9, 1999.

10. Lehman JS, Cook-Norris RH, Weed BR, Weenig RH, Gibson LE, Weaver AL and Pittelkow MR: Folliculotropic mycosis fungoides: Single-center study and systematic review. Arch Dermatol 146: 607-613, 2010.

11. Yost JM, Do TT, Kovalszki K, Su L, Anderson TF and Gudjonsson JE: Two cases of syringotropic cutaneous T-cell lymphoma and review of the literature. J Am Acad Dermatol 61: 133-138, 2009.

12. Kempf W, Ostheeren-Michaelis S, Paulli M, Lucioni M, Wechsler J, Audring H, Assaf C, Rüdiger T, Willemze R, Meijer CJ, et al: Granulomatous mycosis fungoides and granulomatous slack skin: A multicenter study of the Cutaneous lymphoma histopathology task force group of the European organization for research and treatment of cancer (EORTC). Arch Dermatol 144: 1609-1617, 2008.

13. Sarkany I: Patchy alopecia, anhidrosis, eccrine gland wall hypertrophy and vasculitis. Proc R Soc Med 62: 157-159, 1969.

14. Pileri A, Facchetti F, Rütten A, Zumiani G, Boi S, Fink-Puches R and Cerroni L: Syringotropic mycosis fungoides: A rare variant of the disease with peculiar clinicopathologic features. Am J Surg Pathol 35: 100-109, 2011.

15. Santucci M, Biggeri A, Feller AC, Massi D and Burg G: Efficacy of histologic criteria for diagnosing early mycosis fungoides: An EORTC cutaneous lymphoma study group investigation. European organization for research and treatment of cancer. Am J Surg Pathol 24: 40-50, 2000.

16. Hodak E, Feinmesser M, Segal T, Yosipovitch G, Lapidoth M, Maron L, Bergman R, Sahar D and David M: Follicular cutaneous T-cell lymphoma: A clinicopathological study of nine cases. $\mathrm{Br}$ J Dermatol 141: 315-322, 1999.

17. Jacob R, Scala M amd Fung MA: A case of syringotropic cutaneous T-cell lymphoma treated with local radiotherapy. J Am Acad Dermatol 60: 152-154, 2009. 